\title{
Krise der liberalen Demokratie? Nein! Krise des individualistischen Liberalismus
}

\author{
Frauke Höntzsch
}

Online publiziert: 8. April 2020

(C) Der/die Autor(en) 2020

\section{Crisis of Liberal Democracies? No! Crisis of Individualistic Liberalism}

\begin{abstract}
The current crisis of liberal democracy is actually a crisis of individualistic liberalism as recent literature shows by its analysis and more often by its example. Whilst social inequality by many authors is seen as main source of the crisis, it is only the consequence of a contradiction inherent in individualistic liberalism: Equal individual liberty leads to social and in the consequence to political inequality because of the inevitable differences in the use of that individual liberty. However, political equality is a necessary condition of individual liberty as a reciprocal right. Therefore, it is necessary to overcome a purely individualistic understanding of liberty that threatens not only democracy but individual liberty itself.
\end{abstract}

Keywords Liberalism $\cdot$ Liberal democracy $\cdot$ Liberty $\cdot$ Equality $\cdot$ Crisis

Die politischen Systeme des Westens scheinen in der Krise, Populismus und Autoritarismus auf dem Vormarsch. Ausdruck dessen sind zahlreiche, überwiegend im Jahr 2018 erschienene Publikationen, die ausgehend von der Wahl Donald Trumps zum Präsidenten der Vereinigten Staaten von Amerika, dem Votum der Briten für den Brexit sowie dem Erstarken rechtspopulistischer Parteien in fast allen westlichen Demokratien den Ursachen der konstatierten Krise auf den Grund gehen und Auswege aus ihr suchen. Wenn auch viele der Titel die Krise der (liberalen) Demokratie beschwören, wird in der Zusammenschau doch deutlich: Im Kern handelt es sich um eine Krise des Liberalismus, genau genommen eine Krise des zur Ideologie erstarrten individualistischen Liberalismus.

F. Höntzsch (ه)

Universität Augsburg, Augsburg, Deutschland

E-Mail: frauke.hoentzsch@phil.uni-augsburg.de 
Dem rein individualistisch verstandenen Liberalismus, der Freiheit auf die negative Freiheit des Individuums als protection of life and property against force and fraud beschränkt, wohnt von Anbeginn ein Widerspruch inne: die Gefährdung der individuellen Freiheit durch ihre eigenen antiegalitären Effekte. Gleiche individuelle Freiheit erzeugt aufgrund der Unterschiede im Gebrauch dieser Freiheit zwangsläufig ökonomische und soziale und in der Folge politische Ungleichheit und gefährdet so, indem sie das demokratische Versprechen nach gleicher Teilhabe unterminiert, ihre eigenen Voraussetzungen. Ähnlich wie das 19. Jahrhundert vor die ,soziale Frage ' gestellt war, sind wir heute mit einer ,globalen Frage" konfrontiert. Während man das Problem der antiegalitären Effekte individueller Freiheit im nationalen Rahmen durch wohlfahrtsstaatliche Maßnahmen korrigieren (wenn auch nicht lösen) konnte, ist das vor dem Hintergrund globaler Handlungszusammenhänge nicht mehr im selben Maße möglich. Vielmehr tritt angesichts eines entfesselten globalen Kapitalismus immer deutlicher zutage: Der individualistische Liberalismus begünstigt entgegen seiner eigenen Forderung nach gleicher Freiheit paradoxerweise eine elitäre, wenn nicht gar autoritäre Politikvorstellung.

Während die Auswirkungen des ökonomischen (Neo-)Liberalismus und seine Folgen für die Demokratie bereits vielfach thematisiert und kritisiert wurden (in jüngster Vergangenheit besonders prominent etwa von Colin Crouch oder Wendy Brown), so zeigt die neueste Welle von Publikationen gegenüber früheren $\mathrm{Ab}$ handlungen zwei Besonderheiten: Zum einen handelt es sich bei den Verfassern der jüngsten Publikationen überwiegend um Autoren aus dem (sozial-)liberalen bis konservativen Spektrum. Dadurch gerät zum anderen stärker und unmittelbarer der politische Liberalismus in den Blick - allerdings nicht immer auch in die Kritik. Vielmehr ist die Mehrzahl der hier besprochenen Titel selbst (subtiler) Ausdruck der elitären Tendenz des individualistischen Liberalismus, wenn im Fokus des Interesses die Gefährdung der liberalen Errungenschaften durch den Populismus steht, anstatt die Krise des individualistischen Liberalismus (und den Populismus als deren Symptom) ernst zu nehmen. Das ist bedauerlich, insofern eine Kritik des vorherrschenden, politischen Liberalismus überfällig erscheint, auch weil die Kritik am ökonomischen (Neo-)Liberalismus ohne sie unvollständig bleibt. Denn: Der globale Kapitalismus scheint nicht eigentlich Ursache der gegenwärtigen Krise zu sein, sondern vielmehr ,nur' Katalysator für die dem individualistischen Liberalismus inhärenten antiegalitären Effekte, die sich unter seinen Vorzeichen nicht länger durch nationalstaatliche Politik eindämmen lassen.

In einem ersten Überblick lassen sich die hier besprochenen Titel anhand ihrer stärker systematisch-theoretischen beziehungsweise historisch-empirischen Argumentation unterscheiden. Während die systematischen Abhandlungen das Konzept des Liberalismus selbst oder sein Verhältnis zum Konzept der Demokratie in den Blick nehmen, stellen die historischen Studien das Konzept der liberalen Demokratie westlicher Prägung nicht infrage und nehmen entsprechend überwiegend die Symptome der Krise (die sie fälschlicherweise als deren Ursachen benennen) in den Blick.

Das Vorherrschen des (individualistischen) Liberalismus machen nur zwei der systematisch argumentierenden Titel als Ursache der Krise aus: Patrick J. Dene- 
ens „Why Liberalism Failed“1 aus konservativer Sicht und Salvatore Babones „The New Authoritarianism“2 aus soziologischer Perspektive. Zwar benennen auch Yascha Mounk in „,The People vs. Democracy“3 ${ }^{“ 3}$ und William A. Galston in ,AntiPluralism. The Populist Threat to Liberal Democracy“4 das Problem, wenn sie analytisch zwischen den Konzepten der Demokratie und des Liberalismus unterscheiden und eine Entwicklung hin zu illiberalen Demokratien einerseits und zu einem undemokratischen Liberalismus andererseits konstatieren. Ihr Anliegen aber scheint, trotz dieser klaren Analyse, eine einseitige Verteidigung liberaler Werte gegenüber dem Populismus zu sein.

In dieser Parteinahme für den Liberalismus ähneln ihre Ausführungen den (ideen-)historisch oder empirisch argumentierenden Studien ,Democracy and Its Crisis“ von A. C. Grayling Ziblatt ${ }^{6}$ und „The Great Degeneration“"von Niall Ferguson ${ }^{7}$, deren Ziel erklärtermaßen die Rettung der liberalen Demokratie ist. Sie sehen das Problem im Abweichen von der ursprünglichen Form (Levitsky/Ziblatt und Ferguson) beziehungsweise in der mangelhaften Umsetzung der idealen Form der liberalen Demokratie (Grayling). Selbst Kishore Mahbubani tritt mit „Has the West Lost It?" ${ }^{8}$ an, die liberalen Errungenschaften des Westens zu retten, wenn er dem Westen den Rat gibt, seine liberalen Werte auch auf globaler Ebene institutionell auf Dauer zu stellen, bevor seine Macht vollends geschwunden sein wird.

Unter den historischen und empirischen Studien wenden sich einzig „How Democracy Ends“ von David Runciman ${ }^{9}$ und (zumindest dem ersten Anschein nach) auch „The Road to Unfreedom“ von Timothy Snyder ${ }^{10}$ gegen die unterstellte historische Kontinuität und setzen stattdessen auf eine alternative Geschichtsschreibung. Insofern Snyder die Krise aber letztlich in fast verschwörungstheoretisch anmutender Eindimensionalität auf Wladimir Putin zurückführt und Runciman die liberale Demokratie in ihrer Midlife-Crisis sieht und ihr Ende für unabwendbar hält, scheinen sie den eigentlichen Kern der Krise zu verfehlen.

Erwartet man von der versammelten Expertise der vorliegenden Bücher wegweisende Vorschläge für die Überwindung der Krise, wird man enttäuscht. Während die

\footnotetext{
${ }^{1}$ Deneen, Patrick J.: Why Liberalism Failed, Yale UP, New Haven, CT/London 2018.

${ }^{2}$ Babones, Salvatore: The New Authoritarianism. Trump, Populism, and the Tyranny of Experts, Polity Press, Cambridge 2018.

${ }^{3}$ Mounk, Yascha: Der Zerfall der Demokratie. Wie der Populismus den Rechtsstaat bedroht, Droemer Knaur, München, 2018 (engl. 2018).

${ }^{4}$ Galston, William A.: Anti-Pluralism. The Populist Threat to Liberal Democracy, Yale UP, New Haven, CT/London 2018.

5 Grayling, A.C.: Democracy and Its Crisis, Oneworld, London 2017.

${ }^{6}$ Levitsky, Steven/Ziblatt, Daniel: Wie Demokratien sterben. Und was wir dagegen tun können, DVA, München, 2018 (engl. 2018).

${ }^{7}$ Ferguson, Niall: Der Niedergang des Westens. Wie Institutionen verfallen und Ökonomien sterben, List, Berlin, ${ }^{2} 2014$ (engl. 2012)

${ }^{8}$ Mahbubani, Kishore: Hast he West Lost it? A Provocation, Penguin, New York 2018.

9 Runciman, David: How Democracy Ends, Profile Books, London 2018.

10 Snyder, Timothy: Der Weg in die Unfreiheit. Russland, Europa, Amerika, Beck, München, 2018 (engl. 2018).
} 
Beschreibung der Symptome und - in den systematischen Abhandlungen - teils auch der (im individualistischen Liberalismus selbst liegenden) Ursachen ausgesprochen pointiert ist, sind die Vorschläge hinsichtlich möglicher Wege aus der Krise - bis auf wenige Ausnahmen - in der Forderung, den ursprünglichen Zustand der liberalen Demokratie wiederherzustellen, rückwärtsgewandt und auf den Nationalstaat (oftmals in Gestalt der US-amerikanischen Demokratie) beschränkt. Dem entspricht das in den Publikationen vorherrschende Krisenverständnis, das Krise im Sinne des Niedergangs, Verfalls und Verlusts versteht. Dabei scheint in der konstatierten Krise eine historische Chance zu liegen: die Chance, den dem individualistischen Liberalismus von Anbeginn innewohnenden, inhärenten Widerspruch, der in Konflikt mit dem demokratischen Versprechen nach gleicher Teilhabe gerät, zu überwinden. Die momentane Krise fördert diesen Widerspruch überdeutlich zutage und bietet so die Chance, offensichtliche Verkrustungen aufzulösen und das Versprechen der liberalen Demokratie nach gleicher Freiheit, das weder der Liberalismus noch die Demokratie alleine zu garantieren vermag, einzulösen.

\section{Systematische Ansätze}

\subsection{Hat sich der Liberalismus zu Tode gesiegt?}

Lediglich zwei der hier behandelten Titel machen explizit den politischen Liberalismus für die gegenwärtige Krise der politischen Systeme des Westens verantwortlich und unterziehen ihn einer systematischen Kritik: zum einen „Why Liberalism Failed" von Patrick J. Deneen ${ }^{11}$, Professor für Politikwissenschaft an der privaten katholischen University of Notre Dame und zum anderen mit einem populärwissenschaftlichen Anspruch „,The New Authoritarianism. Trump, Populism, and the Tyranny of Experts" des in Sydney lehrenden amerikanischen Soziologen Salvatore Babones. ${ }^{12}$ Beide benennen mit analytischer Schärfe die Ursachen der derzeitigen Krise. Insbesondere Deneen seziert die selbstzerstörerischen Tendenzen des (individualistischen) Liberalismus bis ins Detail; er vergibt aber die Chance, wie von ihm gefordert, ,[to] outgrow the age of ideology“ (S. 183), weil sein Angriff auf den Liberalismus selbst ideologisch motiviert ist.

Deneens These ist zunächst so klar wie überzeugend: „Liberalism [...] has failed because it has succeeded“ (S. 3). Der Liberalismus, so Deneen, sei gescheitert, weil er entgegen seiner Versprechen nach Gleichheit, Pluralismus, menschlicher Würde und Freiheit ,,in practice generates titanic inequality, enforces uniformity and homogeneity, fosters material and spiritual degradation, and undermines freedom“ (ebd.). Anders als die beiden anderen politischen Ideologien - hier nennt Deneen Faschismus und Kommunismus! - sei der Liberalismus besonders heimtückisch, indem er vorgebe, neutral zu sein, dabei aber die Welt heimlich nach seinem Bilde forme. Auf Grundlage einer durch eine falsche Vorstellung der menschlichen Natur determinierten Politik, Ökonomie, Bildung, Wissenschaft und Technologie produziere der

\footnotetext{
11 Deenen: Liberalism (wie Anm. 1).

12 Babones: Authoritarianism (wie Anm. 2).
} 
Liberalismus erst jene vereinzelten, autonomen, bindungslosen und damit zugleich machtlosen, einsamen Monaden, die er voraussetze (S. 16). Um diese zu steuern, fordere und benötige der Liberalismus einen starken Staat (S. 17). Er habe eine neue Aristokratie hervorgebracht, ,that fights ceaselessly to maintain the structures of liberal injustice“ (S. 135) und generiere letztendlich selbst „demotic demands for an illiberal autocrat who promises to protect the people against the vagaries of liberalism itself“" (S. 178).

Deneen lässt keinen Zweifel, dass er eine postliberale Zukunft herbeisehnt. Zwar könne man nicht so tun, als habe das liberale Zeitalter nie stattgefunden und müsse auch die liberalen Errungenschaften nicht leugnen. Diese aber, das wird schnell deutlich, beruhen in Deneens Augen letztlich auf der Kontinuität mit den tiefsten Überzeugungen der westlichen politischen Tradition - nämlich: ,to secure liberty and human dignity through the constraint of tyranny, arbitrary rule, and oppression“und damit auf Werten, die aus der Antike und dem christlichen Mittelalter stammten (S. 19). Die Leistung des Liberalismus schrumpft so verstanden auf dessen Anspruch zusammen, den traditionellen Werten zur Umsetzung zu verhelfen: „Liberalism was a sign of the profound success of the West's most fundamental philosophical commitments, a manifestation of a widespread demand that daily practices should more closely conform to ideals“ (S. 185). Der Liberalismus aber hat dieses Versprechen in Deneens Augen nie eingelöst, ganz im Gegenteil habe er jene Ideale verraten. Grund dafür sei - und das bildet den Kern seiner Kritik am Liberalismus - eine verfehlte Anthropologie, die die traditionellen Errungenschaften unterminiert habe: „Liberalism's break with the past was founded on a false anthropology“ (S. 185). Die liberale Befreiung des Menschen war aus Deneens Perspektive eine Befreiung „from the reality of relational life“ (S. 188), sein erklärtes Ziel ist es demgegenüber, „to [...] build [...] liberty after liberalism“ (S. 198).

Deneens Kritik eines in Selbstwidersprüche verstrickten Liberalismus ist mit Abstand das Erhellendste, was die Flut von Abhandlungen zur derzeitigen Krise der politischen Systeme des Westens hervorgebracht hat; seine Analyse geht über die gegenwärtige Krise hinaus, sie ist auch, das betont der Autor im Vorwort, früher verfasst worden (S. xiii). Doch auch wenn Deneen recht hat und der individualistische Liberalismus (den er verkürzt mit dem Liberalismus gleichsetzt) als gescheitert gelten muss, so doch vor allem aufgrund seiner antiegalitären Effekte, die der Autor zwar benennt, doch zugunsten seines eigentlichen Fokus auf die Wiederbelebung kultureller Normen und lokaler Gemeinschaften völlig vernachlässigt. Weil seine Kritik des Liberalismus letztlich einem ideologischen Programm untergeordnet ist, geht sein Vorschlag für die politische Zukunft an seiner eigenen Analyse vorbei.

So berechtigt und überzeugend die Kritik der individualistischen Anthropologie und ihrer unintendierten Folgen ist, so verstrickt sich Deneen in ideologische Grabenkämpfe, wenn er für den Liberalismus konstatiert, dass er - wie jegliche Ideologie - scheitere, ,because it is based on falsehood about human nature, and hence can't help but fail“", weil früher oder später die Kluft zwischen ideologischen Forderungen und tatsächlichen Erfahrungen der Menschen sichtbar werde (S. 6). Ausdruck von Ideologie ist jedoch nicht erst eine ,falsche" Anthropologie, sondern jeglicher - in Deneens Behauptung einer ,falschen“ Anthropologie zum Ausdruck kommende - Anspruch, über die Erkenntnis der Natur des Menschen zu verfügen 
und aus diesem Wissen, das eine Trennung zwischen Wissenden und Unwissenden impliziert, Herrschaftsansprüche beziehungsweise die ,gute' und ,gerechte ' Ordnung ableiten zu können.

Wie vor diesem Hintergrund nicht anders zu erwarten, liegt Deneens Überlegungen selbst eine substanzialistische (altbekannte) Anthropologie zugrunde: ,the classical and Christian understanding of human beings as fundamentally relational creatures - ,social and political animals“" (S. 185). Und auch wenn Deneen mit Blick auf die politische Zukunft von „tentative first steps“ (S. 182) spricht, leitet er daraus ein sehr konkretes politisches Programm ab: „Out of the fostering of new and better selves, porously invested in the fate of other selves - through the cultivation of cultures of community, care, self-sacrifice, and small-scale democracy a better practice might arise, and from it, ultimately, perhaps a better theory than the failing project of liberalism“ (S. 20). Dem liegt ein Verständnis von Kultur und Tradition zugrunde, dem die postmoderne Annahme der Kontingenz fremd scheint: „Culture and tradition are the result of accumulations of practice and experience that generations have willingly accrued and passed along as a gift to future generations. This inheritance is the result of a deeper freedom, the freedom of intergenerational interactions with the world and one another" (S. 190) und die Annahme, Kultur sei ,the soil in which the human person grows and - if it is a good culture - flourishes“" [Hervorh. d. Verf.] (S. 110). Dass es auch beengende Traditionen und kulturelle Vorschriften waren, gegen deren erzwungene Übernahme sich der Liberalismus wehrte, scheint Deneen nicht zu irritieren: „And as the word [culture] suggests, it is nearly always linked to ,cult", understanding the local to be bound to and ultimately an expression of the universal and eternal, the divine and sublime“" (S. 193).

Vor diesem Hintergrund verkommt auch die moderne Demokratie zur liberalen Täuschung und Verfallserscheinung: ,the adjective [liberal] not only modifies ,democracy ' but proposes a redefinition of the ancient regime into its effective opposite, to one in which people do not rule but are instead satisfied with the material and martial benefits of living in a liberal res idiotica“" (S. 154). Demgegenüber stellt Deneen im Unterkapitel „Illiberal Democracy, Rightly Understood“ unter Berufung auf Alexis de Tocqueville fest, die Praktiken demokratischer Bürgerschaft in Amerika hätten sich bereits vor „America's liberal founding“ entwickelt und ihre Ursprünge lägen ,,in the earlier Puritan roots of the American settlement“, insbesondere im „widely shared understanding of Christian liberty“ (S. 174). Deneen propagiert in der Folge eine zeitgenössische Version dieses antiken und christlichen Freiheitsverständnisses, als dessen Gewährsmann er wiederum Tocqueville sieht: „Tocqueville commends a more contemporary articulation of a classical or Christian notion of liberty of doing what is consonant with the ,just and the good', and not the liberal understanding that defines liberty acting as one likes, as long as no one is physically harmed" (S. 174f.).

Damit unterstellt Deneen nicht nur eine Dichotomie zwischen altem und neuem, positivem und negativem Freiheitsverständnis, die sich in ihrer Einseitigkeit bei Denkern wie Tocqueville gerade nicht findet, vor allem negiert er damit die mit dem modernen Verständnis von repräsentativer Demokratie und liberaler Freiheit untrennbar verbundene Forderung nach politischer Gleichheit. 
So richtig seine Kritik der individualistischen Anthropologie und ihrer unintendierten Folgen, so richtig sein Hinweis auf das daraus folgende verkürzte Freiheitsverständnis und die Forderung nach der Überwindung jeglicher Ideologie ist, so wird doch nicht ersichtlich, warum aus der insuffizienten Umsetzung der liberalen Forderung nach gleicher Freiheit eine christlich inspirierte kommunitäre Politik folgen sollte. Auch wenn Deneen immer wieder und zu Recht anprangert, dass der Liberalismus zu Ungleichheit geführt habe, so scheint Gleichheit für ihn kein zentraler politischer Wert. Die historische Neuerung des Liberalismus aber liegt gerade darin, dass er ,Freiheit und menschliche Würde“ für alle gleichermaßen fordert und dadurch eine ideelle Verbindung mit der Demokratie eingeht. Wenn der Liberalismus in seiner individualistischen Variante dieses Ziel verfehlt, ist zu fragen, wie sich die gleiche Freiheit garantieren und damit der demokratische Aushandlungsprozess schützen lässt.

Salvatore Babones' Perspektive scheint hier folgerichtiger. Auch er identifiziert klar das Vorherrschen der liberalen Ideologie als Problem. Weil er Liberalismus und (moderne) Demokratie aber nicht gleichsetzt, zieht er daraus andere Konsequenzen. Zunächst ähnlich zu Deneens Diagnose konstatiert Babones: „If liberal principles seem threatened, it is only because they have been so successful" (S. 6). Babones sieht einen ,neuen Autoritarismus“ am Werk, der paradoxerweise ein „liberaler Autoritarismus“ sei, eine „Tyrannei der Experten“ (S. 11). Doch wird bereits hier, wenn er nicht vom Scheitern, sondern der Gefährdung liberaler Prinzipien spricht, die andere Perspektive deutlich. Babones sieht das Problem anders als Deneen in einem Ungleichgewicht, genau genommen im Sieg des Liberalismus über den Konservatismus und Progressivismus. Das habe dazu geführt, dass der Liberalismus sich von einer Philosophie individueller Freiheiten in eine Philosophie individueller Rechte entwickelt habe, "that take precedence over those of the democratic polity itself" (S. ix f.). Nur im Falle der Balance der drei Strömungen sei die Vitalität der Demokratie gegeben; Abraham Lincolns oft missverstandenes Diktum bringe das zum Ausdruck: ,Government of the people, of the whole people in a single unified nation, is at the heart of conservatism. Government by the people, ensuring all people their due share in their own government, is at heart of liberalism. And government for the people, for the benefit of the great majority of the people, is at the heart of progressivism“" (S. ix).

Bleibt Babones' Antwort auf die Krise auch dabei stehen und insofern schematisch, so wird seine Fürsprache für die Stärkung der Demokratie gegenüber dem Liberalismus als solche dem beschriebenen Problem doch gerechter als Deneens ideologisches Gegenprogramm. Das scheint auch an der geweiteten Perspektive der Problemdiagnose zu liegen. Babones überwindet - zumindest in seiner Krisendiagnose - als einer der wenigen hier besprochenen Autoren den methodologischen Nationalismus. Er sieht die nationale Demokratie in erster Linie durch den neuen liberalen Autoritarismus der globalisierten Ökonomie und ihrer Experten in Gefahr: „The new liberal authoritarianism, acting at the global level, is perhaps the most dangerous authoritarianism of all [...], dangerous for democracy. Whether we prefer to live in good dictatorship or questionable democracies is (perhaps) for us to decide. But global governance is no democracy. It is private preserve of the global expert class“ (S. 70). Hier scheint Babones einen zentralen Punkt zu benennen. Denn tat- 
sächlich hat sich das Problem der antiegalitären Effekte der individuellen Freiheit im Zuge der Globalisierung zugespitzt. Das Erstarken rechtspopulistischer Parteien kann als Folge dieses immer deutlicheren Zutagetretens des dem individualistischen Liberalismus inhärenten Widerspruchs verstanden werden.

Babones versteht den Populismus vor diesem Hintergrund zu Recht als demokratisches Korrektiv, wenn er auch etwas provokativ von „The Populist Purgative“ spricht und festhält: „Populists are the hooligans who boo the seemingly biased liberal referee“ (S. 103). In der Tat erscheint die Kritik der Populisten berechtigt, auch wenn ihre Antwort verfehlt, weil in sich widersprüchlich ist, insofern die behauptete Trennung zwischen Elite und Volk als je homogene Größen die kritisierte Spaltung vertieft, anstatt sie zu überwinden. Umgekehrt bestätigt aber auch der vorherrschende, liberale Blick auf die Populisten die kritisierte Spaltung, insofern er die Populisten als Gegner der (vernünftigen) liberalen Ordnung als unvernünftig oder wahlweise ungebildet disqualifiziert. Insofern verweist Babones richtigerweise auf den undemokratischen Umgang der liberalen Öffentlichkeit mit dem Populismus: „The democratic principle of one person, one vote is a tonic for humility because it requires politicians to suspend their preconceptions about the intellectual incapacity of ordinary people“ (S. 103). Die Sorgen der, einfachen Leute', mithin die von ihnen gewählten Populisten ernst zu nehmen, hieße in der Tat, sie als gleichwertige Bürger mit gleichwertigen Ansichten zu behandeln.

\subsection{Ursache oder Wirkung? Illiberale Demokratie und undemokratischer Liberalismus}

Beredtes Beispiel für den liberalen Blick auf den Populismus und damit zugleich subtiler Ausdruck der elitären Tendenz des individualistischen Liberalismus sind zwei weitere Titel, die jedoch durch die konzeptuelle Trennung von Demokratie und Liberalismus zugleich für analytische Klarheit hinsichtlich der Ursachen der Krise sorgen: „Anti-Pluralism. The Populist Threat to Liberal Democracy“ von William A. Galston ${ }^{13}$ und „Der Zerfall der Demokratie. Wie der Populismus den Rechtsstaat bedroht" des in Harvard tätigen, deutsch-amerikanischen Politikwissenschaftlers Yascha Mounk. ${ }^{14}$

Die Stärke der beiden Abhandlungen liegt in der analytischen Trennung der Konzepte der Demokratie und des Liberalismus (Galston unterscheidet Demokratie, Liberalismus, Republikanismus und Konstitutionalismus) und der klaren Beschreibung der nur scheinbar konträren Phänomene, Populismus und Elitismus, aus deren Ungleichgewicht. Mounk sieht das Problem der liberalen Demokratie im Verfall ihrer ,einzigartige[n] Mischung aus den Rechten des Individuums und dem Willen des Volkes“ und dem Aufstieg ,,der illiberalen Demokratie, einer Demokratie ohne Rechte, und des undemokratischen Liberalismus, von Rechten ohne Demokratie“ [Hervorh. im Orig.] (S. 24). Galston benennt ganz ähnlich zwei charakteristische Deformationen der liberalen Demokratie, setzt sie aber, anders als Mounk, in ein klares Kausalverhältnis: „Elitists claim that they best understand the means to the

13 Galston: Anti-Pluralism (wie Anm. 4).

14 Mounk: Zerfall (wie Anm. 3). 
public's ends and should be freed from the inconvenient necessity of popular consent. They regard themselves as the defenders of liberal values, but they have doubts about democracy. [...] The result has been liberal democracy's other deformation: the rise of populist movements [...] across the West" [Hervorh. d. Verf.] (S. 4). Galston wie Mounk ziehen jedoch aus dieser klaren Analyse nicht die Konsequenz, nach einer besseren Vereinbarkeit der Konzepte zu fragen. Galston hält die gegenwärtigen Probleme vielmehr für unvermeidbar: „The basic structure of liberal democracy creates tensions that can never be expunged. At best they can be managed in response to ever-changing circumstances" (S. 5f.). Auch Mounk hält die Stabilität der liberalen Demokratie eher für eine Ausnahme, die von äußeren Faktoren abhängig sei (S. 25). Der Grundtenor beider Abhandlungen lautet: Die liberale Demokratie ist durch veränderte Rahmenbedingungen wie Digitalisierung, Masseneinwanderung und wachsende soziale Ungleichheit aus der Balance geraten, weshalb das Ziel sein muss, die Rahmenbedingungen zu verbessern. Die weitestgehende Ausblendung der Rolle des globalen Kapitalismus für die genannten Entwicklungen lässt dabei nicht nur die Lösungsvorschläge fraglich erscheinen, sondern ist umso erstaunlicher, als Populisten mit ihrer Forderung nach Protektionismus, Begrenzung der Einwanderung und Stärkung des Nationalismus gerade die maßgeblich durch den globalen Kapitalismus geprägte liberale Weltordnung in Zweifel ziehen. Anstatt sich mit den hier angesprochenen Problemen auseinanderzusetzen, bestätigen die Ausführungen in ihrer mehr oder weniger klaren Fokussierung auf die Bedrohung liberaler Errungenschaften durch den Populismus in subtiler Weise die elitäre Tendenz des individualistischen Liberalismus.

Besonders deutlich wird das bei Mounk, bei dem schon einleitend unklar bleibt, in welchem Verhältnis die Tendenzen zur illiberalen Demokratie einerseits und zum undemokratischen Liberalismus andererseits zueinander stehen. Mal spricht Mounk von „Zwei Seiten ein und derselben Medaille“ (S. 23), dann wieder scheint er die illiberalen Einstellungen der Bevölkerung für die Entwicklung hin zum undemokratischen Liberalismus verantwortlich zu machen: „Denn während die Bürger zunehmend an liberalen Institutionen und Gepflogenheiten zweifeln, versuchen die politischen Eliten immer stärker, sich von diesem wachsenden Unmut abzuschotten“ (S. 20). Auch im Weiteren scheint Mounk die „gegenwärtige Krise der Demokratie“ mit dem „Aufstieg des Populismus“ gleichzusetzen (S. 158f.) und sein Anliegen primär der „Kampf gegen den Populismus“ (S. 261, 301) und der Schutz der rechtsstaatlichen Freiheit zu sein; darauf deutet nicht zuletzt der Untertitel hin, im Deutschen: „Wie der Populismus den Rechtsstaat bedroht“, wie im Englischen: "Why Our Freedom Is in Danger and How to Save It". Weil Mounk so letztlich Ursache und Wirkung der Krise verwechselt, doktert er mit Blick auf seine Lösungsvorschläge überwiegend und wenig zielführend an den Symptomen herum. Mounk sieht, klassisch sozialdemokratisch, die wirtschaftliche Stagnation und die damit einhergehenden Abstiegsängste als Grund für die Krise, verschärft durch den Siegeszug des Internets und der sozialen Netzwerke sowie durch die Auflösung monoethischer Nationalitäten. Entsprechend seiner Krisendiagnostik empfiehlt Mounk den Nationalismus zu zähmen, die Wirtschaft zu sanieren und den Glauben an die Demokratie zu erneuern: Er will die „wachsende Ungleichheit eindämmen“, in den Vordergrund stellen, was die Mitglieder einer multiethischen Demokratie „eint, an- 
statt unentwegt zu betonen, was sie voneinander trennt“ und lernen ,viel besser mit dem transformativen Einfluss des Internets und der sozialen Medien zurechtzukommen“" (S. 28). So zustimmungswert das alles klingt, so scheint es angesichts globaler Handlungszusammenhänge nicht nur naiv, sondern darüber hinaus Besitzstandswahrung. Mounks Forderung, ,die Sorgen der einfachen Leute ernst zu nehmen - und ihre Sprache zu sprechen“ (S. 219), offenbart einen subtilen liberalen Elitismus, dessen Problem vor allem in einem Vermittlungsproblem zu bestehen scheint, weil es, scheinbar zu Mounks Bedauern, nicht mehr ,einer kleinen politischen und finanziellen Elite vorbehalten [ist], mit den Massen zu kommunizieren“ (S. 26). Durch das Internet sei die Machtbalance verschoben worden: „Auch deshalb scheinen die Unruhestifter immer mehr die Oberhand gegenüber den Kräften der Kontinuität zu gewinnen“ (S. 27). Problematisch aber scheint nicht die Öffnung des demokratischen Diskurses, sondern dessen Verunmöglichung durch die gezielte Nutzung technischer Möglichkeiten zur Manipulation von Tatsachen, Etablierung geschlossener Parallelöffentlichkeiten und anonymer Hetze beziehungsweise Einschüchterung Andersdenkender.

Auch Galston sieht als hauptsächliche, gleichwohl ausdrücklich nicht alleinige Ursache für die Krise die wirtschaftliche Stagnation und die damit verbundenen Ängste; dem liegt ein erstaunlich undemokratisches Verständnis der liberalen Demokratie zugrunde, wonach diese auf einem stillschweigenden Übereinkommen zwischen dem Volk und den gewählten Repräsentanten sowie den nicht gewählten Experten (!) beruhe; das Volk füge sich den Eliten, solange anhaltender Wohlstand und stetige Verbesserungen des Lebensstandards gewährleisten seien (S. 2). Dieses Übereinkommen nun sieht Galston nicht nur durch die wirtschaftlichen Entwicklungen, sondern in jüngerer Zeit auch durch Fehler im Umgang mit den ,waves of immigration“ sowie neuer, auf Bildungsunterschieden beruhender kultureller Spaltungen gefährdet (S. 3). Obwohl Galston, anders als Mounk, den Populismus eigentlich explizit als Folge der undemokratischen Tendenzen der liberalen Eliten versteht, zeigen seine Ausführungen, dass er die Trennung zwischen einer im Urteil überlegenen Elite und dem Volk, das seine langfristigen Interessen zu erkennen nicht selbstständig in der Lage ist, als solche nicht infrage stellt: „Liberal democracy needs leaders who eschew the extremes of populism and elitism. [...]. Their task is to find effective means for achieving public goals while helping the people better understand their long-term interests“ (S. 6). Vielmehr ist die Krise für Galston letztlich eine Krise der „,demokratischen Führung“. Die Lösung liegt entsprechend in guter ,demokratischer Führung“, die einen Mittelweg finden müsse zwischen ,leadership and democratic humility“ (S. 121), die Fähigkeit und Legitimität kombinieren müsse: ,they have the attributes needed to exercise power wisely while respecting the ongoing need for public authorization“ (S. 117). Vor diesem Hintergrund scheinen die gegenwärtigen Probleme handhabbar: „Public policy can mitigate the heedlessness of markets and slow unwanted change. Nothing requires democratic leaders to give the same weight to outsiders' claims as to those of their own citizens. [...] Moderate self-preference is the moral core of defensible nationalism. Unmodulated internationalism will breed - is breeding - its antithesis, an increasingly unbridled nationalism“ (S. 5).

$\mathrm{Ob}$ das wirklich trägt, daran aber scheint Galston selbst Zweifel zu hegen, den die Essaysammlung schließt mit einem Kapitel über die „Incompleteness of Libe- 
ral Democracy“, das sich wie eine Sammlung möglicher Probleme und Antworten liest. Hier kritisiert er unter anderem die liberale Anthropologie als verkürzt, bemängelt die Unterdrückung der menschlichen Veranlagung zum Tribalismus durch die liberale Demokratie, thematisiert die ungelöste Ambivalenz von Freiheit und Gleichheit, von Hierarchie und Gleichheit sowie die Spannung zwischen Staat und Markt; gleichwohl diese Punkte unsystematisch nebeneinanderstehen, sind hier doch zentrale Punkte des inhärenten Widerspruchs des individualistischen Liberalismus und der aus ihm resultierenden Probleme angesprochen. So konterkariert Galston hier in letzter Konsequenz sein eingangs formuliertes Verständnis der liberalen Demokratie als ein Übereinkommen zwischen Bürgern und Eliten zur Wohlstandssicherung und Steigerung des Lebensstandards als ein die Probleme nur verdeckendes, aber nicht lösendes Konstrukt.

Die in beiden Abhandlungen nur subtil zum Ausdruck kommende elitäre Tendenz des individualistischen Liberalismus zeigt sich besonders deutlich an dessen äußersten Rand, im libertären Diskurs, wo etwa Jason Brennan in seinem Buch „Gegen Demokratie. Warum wir die Politik nicht den Unvernünftigen überlassen dürfen" 15 die Herrschaft der rationalen Elite fordert und zu dem Schluss gelangt, „dass manche Bürger [...] kein Wahlrecht oder ein im Vergleich zu anderen Bürgern eingeschränktes Wahlrecht haben sollten“ (S. 8) und das obwohl er sich nach eigenem Bekunden für das Wahlrecht interessiert, ,weil dieses politische Recht anders als jene Rechte, die ich als bürgerliche oder wirtschaftliche Freiheitsrechte bezeichne - in erster Linie das Recht beinhaltet, Macht über andere auszuüben“ [Hervorh. im Orig.] (S. 28). Das aber spricht gerade für die gleiche Freiheit eines jeden, es sei denn, man geht von der Existenz eines höheren Wissens aus, das nur einige Wenige innehaben. In der Forderung nach der Herrschaft der Kompetenten treffen sich libertäre und autoritäre Vorstellungen.

\section{Historisch-empirische Analysen}

\subsection{Back to the Roots}

Einen anderen Ausgangspunkt wählen die (ideen-)historisch beziehungsweise empirisch argumentierenden Titel von A. C. Grayling „Democracy and Its Crisis“"16, von Steven Levitsky und Daniel Ziblatt „Wie Demokratien sterben. Und was wir dagegen tun können“17 und der bereits 2012 erschienene Band von Niall Ferguson „,Der Niedergang des Westens. Wie Institutionen verfallen und Ökonomien sterben“. ${ }^{18}$ Diesen Büchern liegen ähnliche Annahmen zugrunde: Die liberale Demokratie ist vom richtigen Weg abgekommen (Levitsky/Ziblatt), degeneriert (Ferguson) oder wurde nicht richtig umgesetzt (Grayling) und soll durch die (Wieder-)Herstellung

\footnotetext{
15 Brennan, Jason: Gegen Demokratie. Warum wir die Politik nicht den Unvernünftigen überlassen dürfen, Ullstein, München, 2017 (engl. 2016).

16 Grayling: Democracy (wie Anm. 5).

17 Levitsky/Ziblatt: Demokratien (wie Anm. 6).

18 Ferguson: Niedergang (wie Anm. 7).
} 
einer ursprünglichen beziehungsweise idealen Form gerettet werden. So wollen Levitsky und Ziblatt nicht nur zeigen, „Wie Demokratien sterben“, sondern - wie der programmatische Untertitel verrät - auch „,was wir dagegen tun können“; Grayling eröffnet seine Ausführungen mit einem klaren Bekenntnis: „This book is about the failure of the best political system we have: democracy. And it is about how to put it right“ (S. ix). Ferguson ist überzeugt, ,dass wir, solange wir das wahre Wesen unserer institutionellen Degeneration nicht erkannt haben, unsere Zeit damit verschwenden, mit Quaksalbermitteln an Symptomen herumzudoktern“ (S. 29). Insofern die Abhandlungen das Problem im Abweichen von einer (empirisch oder theoretisch) funktionierenden Form sehen, die es (wieder-)herzustellen gelte, wird die Möglichkeit eines der liberalen Demokratie inhärenten konzeptuellen Konstruktionsfehlers von Anfang an ausgeschlossen, das heißt der Widerspruch, der der liberalen Demokratie individualistischer Prägung innewohnt, wird übersehen und das Problem auf die mangelhafte Umsetzung reduziert. Die Stärke dieser Titel liegt in der Folge in der Beschreibung der Symptome der Krise (die sie gleichwohl als Ursachen ausweisen), sei es aus ideengeschichtlicher (Grayling), aus systemvergleichender (Levitsky/Ziblatt) oder aus ökonomischer Perspektive (Ferguson).

A. C. Graylings Ansatz hebt sich von den beiden anderen ab, insofern zwar auch er die britische und US-amerikanische Demokratie mit einer als ideal verstandenen Form kontrastiert, aber nicht den Verfall einer historischen Umsetzung der Demokratie, sondern die mangelhafte Umsetzung des theoretischen Konzepts kritisiert. Er hat folglich durchaus einen systematischen Anspruch und kommt aus einer ideengeschichtlichen Perspektive zu einem ähnlichen Ergebnis wie Mounk und Galston. Grayling setzt bei Platon an und zeichnet die Geschichte der Demokratie als Geschichte eines Dilemmas, das erst die Federalists im revolutionären Amerika sowie Benjamin Constant, Alexis de Tocqueville und John Stuart Mill in Europa lösen konnten: den Herrschaftsanspruch der Vielen zu realisieren und dabei die Gefahr des Kippens in eine Ochlokratie oder versteckte Oligarchie zu verhindern (S. 3). Er benennt damit letztlich eben jene beiden Tendenzen beziehungsweise Deformationen, die auch Mounk und Galston thematisieren, hält dieses Dilemma konzeptuell aber durch die Denker des 19. Jahrhunderts für bereits gelöst. Sie hätten, so Grayling, beide Seiten, ,that the ultimate source of political authority should lie in democratic assent, and that government should be and could be sound and responsible“ (S. 5), versöhnt. Die Lösung des demokratischen Dilemmas liege in der republikanischen oder repräsentativen Demokratie. Sie sei nur nicht richtig umgesetzt worden: ,[T]he solution to the dilemma of democracy proposed by the thinkers earlier discussed is right, and will work if implemented properly, vigorously, clearly, and fully“ (S. 153). Die heutigen repräsentativen Demokratien in Großbritannien und den USA aber, so Graylings These, „ha[ve] been made to fail“ (S. ix). Grayling sieht dafür drei Hauptgründe, die er in seinen Ausführungen für beide Systeme zu belegen versucht: Erstens nutzten die Regierungsverantwortlichen die Institutionen für ihre eigenen oder parteipolitische Zwecke, zweitens mangele es an politischer Bildung der Wählerschaft und drittens würden beide Gruppen manipuliert durch ,,agencies with partisan interests who recognize that they are unlikely to get their interests favoured in mainstream ways otherwise, and who therefore resort to undemocratic means to get the democracy to deliver their preferred outcomes“ (S. 132). 
Grayling verweist mit allen Punkten letztlich auf das Problem der Dominanz partikularer Interessen in politischen Entscheidungen und damit - auch wenn er das selbst so nicht benennt - auf das zentrale Moment der Gefährdung der individuellen Freiheit durch ihre eigenen antiegalitären Effekte. Denn indem sich die durch die unterschiedliche Nutzung der Freiheit entstehenden Ungleichheiten in politische Entscheidungen übersetzen, weil Partikularinteressen über kollektive Angelegenheiten entscheiden, wird letztlich die individuelle Freiheit, als deren Garant die gleiche Teilhabe in der liberalen Demokratie dient, gefährdet. Grayling rekurriert insofern auch zu Recht auf eben jene Denker, die den Einfluss selbstsüchtiger Interessen auf die Politik zu begrenzen suchten. So richtig jedoch die Problemstellung ist, nämlich die liberale Demokratie vor einer Tyrannei der Mehrheit ebenso wie vor einer versteckten Oligarchie zu schützen, und so richtig die Diagnose der Dominanz politischer Entscheidungen durch partikulare Interessen, so bedauerlich ist, dass Grayling mit Blick auf die Überwindung der Krise mit den liberalen Denkern des 19. Jahrhunderts auf die Kraft der Bildung setzt. Gleichwohl er auch zielführende praktische Vorschläge, wie Transparenz hinsichtlich Wahlkampfspenden, unterbreitet (S. 186f.), übersieht er nicht nur die zentrale Rolle globaler Handlungszusammenhänge für die Dominanz vornehmlich ökonomischer Interessen im politischen Entscheidungsprozess. Durch die starke Betonung der civic education übernimmt er auch die problematische anthropologische Prämisse einer höheren Natur des Menschen, die es unter anderem im Rahmen politischer Beteiligung zu entwickeln gelte - und mit ihr die darin enthaltene elitäre Tendenz, die sich auch im aristokratischen Liberalismus Tocquevilles und Mills zeigt und impliziert, dass man die Bürger nur richtig bilden müsse, damit sie die richtigen Entscheidungen treffen. Das ist umso bedauerlicher, als sich etwa bei Mill durchaus auch von der Prämisse der Entwicklungsfähigkeit des Menschen unabhängige freiheitstheoretische Erwägungen finden ließen, die auf den Schutz der politischen Zusammenarbeit vor individueller Willkür zielen.

Anders als Grayling sehen Levitsky und Ziblatt (wie auch Ferguson) das Problem weniger in der mangelhaften praktischen Umsetzung des richtigen theoretischen Konzepts als in der Degeneration der ursprünglich funktionierenden, empirischen Demokratie. Steven Levitsky und Daniel Ziblatt, Professoren für Regierungslehre in Harvard, treten an, ihre Expertise aus jahrelanger Demokratie- und Autoritarismusforschung zu nutzen, um vor dem schleichenden Verfall der amerikanischen Demokratie zu warnen. Als Referenz dienen dabei nicht Fälle wie die Machtergreifung Augusto Pinochets in Chile, sondern die Politik von Hugo Chávez in Venezuela: Demokratien, so die Autoren, sterben nicht mehr ganz plötzlich durch Waffengewalt, sondern schleichend durch die Aushöhlung ihrer Normen und Institutionen, nicht durch Generäle und Soldaten, sondern durch gewählte Führer: „Der demokratische Rückschritt beginnt heute an der Wahlurne“ (S. 13). Im historischen Vergleich soll ein Muster sichtbar werden, auf dessen Grundlage die Autoren einen „Lackmustest“ entwickeln, der die „Möchtegern-Autokraten“ zu erkennen und im besten Fall von der Macht abzuhalten oder, so sie bereits an die Macht gelangt sind, ihnen wirksam entgegenzutreten hilft (S. 15). Zentraler Akteur sind auch hier die politischen Eliten beziehungsweise besonders die Parteien: „Obwohl es auch wichtig ist, wie die breite Masse auf extremistische Verlockungen reagiert, ist es doch wichtiger, ob politische 
Eliten, und insbesondere Parteien, als Filter dienen. Einfach gesagt, Parteien sind die Wächter der Demokratie“ (S. 30). Abseits der hier zum Ausdruck kommenden Schwäche der affirmativen Argumentation, die blind für die konzeptuellen Gründe der Krise ist und einmal mehr die elitäre Tendenz eines individualistischen Liberalismus offenbart, scheint die Betonung demokratischer Normen für das Funktionieren einer liberalen Demokratie überzeugend, die die Autoren als ,"weiche Leitplanken“ (S. 120) bezeichnen. Die amerikanische Verfassung wurde demnach lange Zeit durch zwei grundlegende Normen geschützt, durch ,gegenseitige Achtung“ und ,institutionelle Zurückhaltung“ (S. 120 ff.), deren Erosion in den 1980er und 1990er Jahren begann und durch Trump nur beschleunigt wurde: „Die Forderung, Konkurrenten als legitime Mitbewerber um die Macht zu betrachten und seine institutionellen Rechte im Geist des Fairplay nicht voll auszuschöpfen, steht nicht in der Verfassung. Doch ohne diese Normen funktioniert die in der Verfassung verankerte Gewaltenteilung und gegenseitige Kontrolle nicht so, wie wir es erwarten“ (S. 249).

Die Ansätze zur Überwindung der Krise beziehen sich wie schon die Ursachensuche - die Rolle des globalen Kapitalismus für die Krise nationaler Demokratien weitestgehend ausblendend - überwiegend auf die USA und setzen entsprechend der Annahme der zentralen Rolle der Parteien bei deren Reform an. Vor allem die republikanische Partei, die die Autoren hauptverantwortlich für die politische Spaltung machen, müsse sich reformieren, wobei ausgerechnet die Erneuerung der CDU nach 1945 als Beispiel dient (S. 263 ff.). Doch auch bei den Demokraten sehen die Autoren Handlungsbedarf: „Absolut notwendig ist jedoch, so schwierig es auch sein mag, dass die Demokraten das Thema der Ungleichheit aufgreifen, denn es betrifft nicht nur die soziale Gerechtigkeit: Das Wohl unserer Demokratie hängt davon ab“ (S. 270). Das Problem der extremen Spaltung des Landes, der Polarisierung, der Feindschaft der politischen Lager führen die Autoren auf eine Verbindung aus sozialer Ungleichheit mit der ,Rassenfrage“ (S. 267 f.) zurück. Wie Mounk und Galston sehen auch Levitsky/Ziblatt einen Großteil der Lösung in sozialstaatlichen Maßnahmen, hier „nach dem Vorbild der skandinavischen Länder“ (S. 269). Das scheint angesichts der US-amerikanischen politischen Kultur nicht nur sehr optimistisch, sondern blendet auch aus, dass Länder mit einem umfassenden Sozialstaat vor ähnliche Probleme gestellt sind, was nicht zuletzt auch die isolierte Behandlung der Krise als einer Krise des US-amerikanischen Systems fragwürdig erscheinen lässt. Und auch wenn Levitsky und Ziblatt am Ende mit Blick auf die neuen Herausforderungen festhalten, nur die liberalen Ideale einer vergangenen Epoche zu erneuern, reiche nicht aus, bleibt doch Zukunftsmusik, wie es gelingen soll, ,Demokratie und ethnische Vielfalt miteinander zu vereinen“" (S. 272).

Auch Niall Ferguson, Historiker mit Schwerpunkt Finanz- und Wirtschaftsgeschichte in Harvard, will durch eine Rückbesinnung auf die liberale Tradition die liberale Demokratie westlicher Prägung (und die damit in seinen Augen verbundene westliche Vorherrschaft) retten. „Der Niedergang des Westens. Wie Institutionen verfallen und Ökonomien sterben" ${ }^{19}$ ähnelt hinsichtlich der angenommenen Degeneration des ehemals erfolgreichen politischen Systems der Analyse von Levitsky/ Ziblatt. Das vor dem Hintergrund der Finanzkrise verfasste Werk fokussiert dabei

19 Ferguson: Niedergang (wie Anm. 7). 
stärker auf die ökonomische Dimension der Krise, jedoch bleibt auch hier - und hier besonders irritierend - der Blick auf den Nationalstaat, mithin nationale Ökonomien, beschränkt. Ausgangspunkt von Fergusons Überlegungen ist die wirtschaftliche Stagnation im Sinne Adam Smiths ,,Stadium des Stillstands“, [...] jenen Zustand eines vormals reichen Landes, das aufgehört hat zu wachsen“" (S. 17), dessen Ursache Ferguson im Anschluss an Smith in den heruntergekommenen Institutionen und Gesetzen sieht: „Die Große Rezession ist bloß ein Symptom einer tiefgreifenden Großen Degeneration“ (S. 19). Demokratie, Kapitalismus, Rechtstaat und Zivilgesellschaft als komplexe Ansammlungen von Institutionen in den Bereichen Politik, Wirtschaft, Recht und Gesellschaft waren in diesem Verständnis entscheidend für den Erfolg des Westens, weil nur eine gute Kombination von Institutionen die richtigen Anreize setze (wobei der Begriff der Institutionen sehr weit gefasst ist und entsprechend vage bleibt (S. $21 \mathrm{f}$.)). Unter Rückgriff auf Klassiker des konservativen Liberalismus versucht Ferguson die Degeneration der Institutionen zu verdeutlichen und Wege aus der Krise zu weisen: Er sieht die Staatsschulden als Symptom für die Auflösung des Burke'schen Gesellschaftsvertrags zwischen den Generationen (S. 59), spricht sich mit Walter Bagehot gegen komplexe staatliche Marktregulierung aus (S. 90f.), sieht die Herrschaft des Rechts zu einer Herrschaft der Rechtsanwälte verkommen (S. 124) und fordert mit Tocqueville eine Stärkung der Zivilgesellschaft durch weniger Staatstätigkeit und mehr Eigeninitiative (S. 139ff.). Wie er sich das vorstellt, verdeutlicht er anhand einer Anekdote einer von ihm ins Leben gerufenen Strandsäuberungsaktion (S. $127 \mathrm{ff}$.) und der Forderung nach Privatisierung der Schulen (S. 142ff.). Ferguson will vor allem die Augen öffnen: Der „stationary state“, die Schulden, die wachsende Ungleichheit sind auf die degenerierten Institutionen zurückzuführen und dementsprechend zu lösen. Den Schlüssel dafür sieht er in der Zivilgesellschaft: „Unsere Aufgabe in den kommenden Jahren ist es, sie [die Institutionen] wiederherzustellen - die Große Degeneration umzukehren - und jene Grundprinzipien einer wahrhaft freien Gesellschaft wieder zum Tragen zu bringen, für die ich - mit Hilfe einiger großer Denker der Vergangenheit - in den vorangegangenen Kapiteln geworben habe. Kurz, es ist Zeit, den Strand zu säubern“ (S. 152). Ob Fergusons Fürsprache für die big society und damit der Versuch, gleichzeitig größere individuelle Freiheit zu ermöglichen und mehr Gemeinsinn zu schaffen, zielführend sind, scheint vor dem Hintergrund der antiegalitären Effekte individueller Freiheit mehr als fraglich. Einem solchen Denken liegt die Annahme zugrunde, individuelle Freiheit schaffe unintendiert sozialen Mehrwert. Diese Annahme scheint in der gegenwärtigen Krise jedoch eher Teil des Problems als Teil der Lösung zu sein.

Die Diagnose des wirtschaftlichen Niedergangs des Westens steht auch im Mittelpunkt von „Has the West Lost It? A Provocation“ von Kishore Mahbubani, Politikwissenschaftler und vormals Singapurs Botschafter bei der $\mathrm{UN}^{20}$, der das Übergewicht des Liberalismus bereits als gegeben hinzunehmen beziehungsweise zu befürworten scheint. Obgleich seine Perspektive eine Außenperspektive ist und Mahbubani den Niedergang des Westens, anders als Ferguson, für unvermeidlich hält und begrüßt, bewertet er die Entwicklung dennoch aus einer rein ökonomischen Fortschrittslogik heraus und setzt auf einen Sieg der so verstandenen westlichen

\footnotetext{
20 Mahbubani: West (wie Anm. 8).
} 
neoliberalen Utopie, wenn auch nicht durch westliche Dominanz und - in seinem Verständnis des Liberalismus als Neoliberalismus konsequent - nicht notwendig in Verbund mit Demokratie.

Mahbubanis Pamphlet ist ein Ratgeber in machiavellistischer Manier, adressiert an den Westen. Der Autor rät, den Tatsachen ins Auge zu blicken und das Beste aus der Situation zu machen: Der ökonomische und in der Folge politische Niedergang des Westens beziehungsweise seiner Vormachtstellung ist unvermeidlich, seine Dominanz ohnehin nur ein historischer Wimpernschlag, aber er könne die verbleibende Macht nutzen, um machiavellistisch seine Utopie zu retten. Der Westen habe dem Rest ein Geschenk gemacht, ,the power of reasoning“ (S. 11), das zunächst in den asiatischen Gesellschaften drei stille Revolutionen ausgelöst habe (und nun durch deren Vorbild auf Afrika und Lateinamerika ausstrahle), weshalb die Entwicklung in vielen Länder sehr viel positiver sei als der pessimistische Blick des Westens suggeriere. Die politische Revolution hat demnach dazu geführt, dass die meisten asiatischen Führer realisiert hätten, dass sie ihrem Volk verantwortlich seien, auch wenn es keine demokratische Mitbestimmung gebe (das scheint für Mahbubani aber verzichtbar, solange der Lebensstandard steigt) (S. 12 ff.); die psychologische Revolution liege im Glauben an eine bessere Zukunft (S. $15 \mathrm{f}$.) und die dritte Revolution habe unter den gegenwärtigen politischen Führern zur Überzeugung geführt, ,that good governance will transform and uplift their societies“ (S. 17). Mahbubani mahnt, diese Erfolge (die er fast ausschließlich an wirtschaftlicher Prosperität misst) nicht zu verspielen, der Westen sei der Verwirklichung seiner Utopie näher denn je (hier führt er als Beweis den Rückgang von Gewalt und Armut an). Mahbubani rät dem Westen, sich von seinem aggressiven Interventionismus zu verabschieden und sich multilateralen Regeln zu unterwerfen, um die Standards festzulegen für die Zeit nach der westlichen Dominanz: „A simple dose of Machiavelli is what we need to save the West and the Rest. Otherwise, the West really has lost it“" (S. 91).

\subsection{Geschichte ist, was wir draus machen}

Als Weckruf verstehen sich auch zwei weitere historisch argumentierende Titel, die sich von den übrigen historisch argumentierenden Abhandlungen darin unterscheiden, dass sie die geschichtliche Kontinuität infrage stellen und die Krise in der Folge als Chance für einen Neuanfang verstehen. Während der Historiker Timothy Snyder mit „Der Weg in die Unfreiheit. Russland - Europa - Amerika“"21 in erster Linie die Zwangsläufigkeit der jüngeren Geschichte in Zweifel ziehen möchte und letztlich doch wieder auf eine Wiederbelebung der liberalen Demokratie zu hoffen scheint, bricht David Runciman, Politikwissenschaftler aus Cambridge, in „How Democracy Ends“ radikaler mit der liberalen Demokratie. ${ }^{22}$ Runciman stellt mit der Annahme, die liberale Demokratie werde wie jede andere Regierungsform enden, die suggerierte Zwangsläufigkeit der Wiederherstellung des liberalen Ideals infrage.

Snyder will die Gegenwart für die Geschichte und damit die Geschichte für die Politik zurückgewinnen. Seine These: Die USA und Europa haben sich mit der in

\footnotetext{
21 Snyder: Weg (wie Anm. 10).

22 Runciman: Democracy (wie Anm. 9).
} 
Anschluss an die Erzählung vom Ende der Geschichte durchsetzenden „Politik der Unausweichlichkeit" - deren positiven Zukunftsprognosen sich angesichts der Finanzkrise 2008, wachsender Ungleichheit und fehlender sozialer Absicherung als hinfällig erwiesen haben - für die von Russland propagierte „Politik der Ewigkeit“ anfällig gemacht (S. 14ff.). Beide schaffen laut Snyder Geschichte ab (S. 16). Snyders Ziel ist es, durch die Analyse der neueren Geschichte deutlich zu machen, dass Unausweichlichkeit und Ewigkeit, Fortschritt und Verhängnis nicht Geschichte selbst sind, ,sondern Ideen innerhalb der Geschichte, Erfahrungsmöglichkeiten in unserer Zeit, die ihre Trends beschleunigen, während sie unser Denken verlangsamen“ (S. 293). Dagegen sei Geschichte „,das, was wir selbst tun“ (ebd.). Der Autor will entlarven und ermächtigen, zeigen, es liegt in unserer Hand: „Eine Geschichte des Verlustes wird so zum Vorschlag für einen Wiederaufbau“ (ebd.).

So erfrischend Snyders Grundaussage ist, sein (zunächst scheinbar) emanzipatives Verständnis von Geschichte angesichts einer schneller werdenden, ständig unter Handlungsdruck stehenden und deshalb scheinbar alternativlosen Politik und so richtig prinzipiell auch die Weitung des Blicks über den Nationalstaat hinaus ist, so schrammt das durchaus spannend zu lesende und faktenreiche Buch in seiner einseitigen Rückführung allen Übels auf Putin und dessen Prägung durch Iwan Iljin hart an der Grenze zur Verschwörungstheorie vorbei. Snyders Blick in die jüngere russische, ukrainische, europäische und amerikanische Vergangenheit soll helfen, die politischen Probleme der Gegenwart zu enthüllen; letztlich schreibt er aber eine Geschichte der wachsenden russischen Einflussnahme auf den Westen, in dramaturgischer Abfolge beginnend mit Putins Wahl des faschistischen Philosophen Iwan Iljin zur ideologischen Leitfigur, über den Zerfall der demokratischen Politik in Russland, Russlands Angriff auf die EU, die russische Invasion in der Ukraine, den russischen Informationskrieg in Russland, Europa und Amerika und schließlich gipfelnd in der von Russland beeinflussten Wahl Donald Trumps. Am Ende dieses ,Weges in die Unfreiheit' steht die Hoffnung auf die Wiederauferstehung der liberalen Demokratie aus den ,Tugenden der Gleichheit, Individualität, Nachfolge, Integration, Neuerung und Wahrheit“. Die zwar laut Snyder allesamt ,,von menschlichen Entscheidungen und Handlungen abhängen“ (S. 293), aber dann doch eine Zwangsläufigkeit suggerieren, die der vorausgegangenen Kritik zu widersprechen scheint: „Die letztgültige Wahrheit ist in dieser Welt unerreichbar, aber das Streben nach ihr führt das Individuum von der Unfreiheit fort. [...] Der Autoritarismus beginnt, wenn wir den Unterschied zwischen dem Wahren und dem Gefälligen nicht mehr benennen können“ (S. 294).

David Runciman dagegen betont den offenen Ausgang der gegenwärtigen Entwicklungen. Er bestreitet ausdrücklich jegliche historische Analogie: „History does not go into reverse“ (S. 4); vor allem findet er, ,we ought to get away from our current fixation with the 1930s" (S. 8f.). Er sieht drei fundamentale Unterschiede der derzeitigen Situation zu früheren Krisen der liberalen Demokratie: Erstens sei die politische Gewalt zurückgegangen, ein Coup unwahrscheinlich, vielmehr werde die Demokratie von innen ausgehöhlt, Symptom hierfür sei die Verbreitung von Verschwörungstheorien (Kap. 1); zweitens hätten Katastrophen wie nukleare Kriege und die Erderwärmung früher zu kollektiver Mobilisierung geführt, erzeugten heute dagegen Passivität (Kap. 2); drittens höhlten die Effekte der digitalen Revolution die 
Demokratie auf vielfältige Weise aus (Kap. 3). Der Autor sieht das Problem in der Folge seiner Diagnose in einer Erschlaffung der Kräfte; die Demokratie befinde sich in ihrer Midlife-Crisis, deren Ausgang offen sei (S. 5). Auch Runciman lässt sich als Weckruf lesen: Wir haben die Zukunft in der Hand; auch wenn die westlichen Demokratien ihren Zenit überschritten und ihre Fähigkeit, sich selbst wiederzubeleben, eingebüßt haben, sollten wir nach vorne schauen: „Western democracy will survive its mid-life crisis. With luck, it will be a little chastened by it. It is unlikely to be revived by it. This is not, after all, the end of democracy. But this is how democracy ends" (S. 218). So sinnvoll Runcimans Hinweis auf die Grenzen des Vergleichs mit früheren Krisen ist, so sprechen nicht erst die weltweiten Klimaproteste gegen seine Diagnose; wie andere transnationale Protestbewegungen (und auch der Populismus) scheinen sie gerade Zeichen einer Wiederbelebung der Demokratie gegen deren (neo-)liberale Unterminierung durch die globalisierte Ökonomie.

Runciman aber ist nicht nur überzeugt, dass ,there are better options than contemporary democracy“, seine Hoffnung ruht in einer ,liberated technology“ (S. 205); die digitale Technologie eröffne die Chance, die politische Organisation zu revolutionieren. Er folgt hier Philip N. Howards Vision aus „Pax Technica“: „He envisages two levels of politics in the future. [...] It leaves technical governance on one side and direct political action on the other. There is little need for anything in between" (S. 197 f.). Runciman behauptet keine Zwangsläufigkeit der Entwicklung: „It includes all sorts of potential futures: some wondrous, some terrible, and most wholly unknowable“ (S. 205). Man kann dem folgen oder nicht, erfrischend ist Runcimans Bereitschaft, auf etwas Neues zuzugehen und die damit einhergehende Beschreibung der Gefühlslage im vorherrschenden Krisendiskurs. Es bleibe nichts anderes übrig, als es zu versuchen, das aber sei besser, als sich in der Vergangenheit und dem Verlust zu suhlen: „To get the best possible future we have to run the gauntlet of the worst [...]. The democracy that many have grown to dislike and distrust remains a comfortable and familiar place to be, compared with the prospect of the unknown. This is our mid-life crisis. We may prefer to wallow on it" (S. 206).

\section{Freiheitliche Demokratie ohne Ideologie}

Die derzeitige Krise der liberalen Demokratie gründet in der Dominanz des liberalen über das demokratische Element, das zeigen die hier besprochenen Titel durch ihre Analyse und/oder noch häufiger durch ihr Beispiel. Während die Beschreibung der Symptome und zum Teil auch der Ursachen der Krise überzeugt, sind die Ansätze zur Überwindung entweder - in Folge einer nur oberflächlichen Diagnose - rückwärtsgewandt und auf den Nationalstaat bezogen oder ziehen - aus (je unterschiedlichen) ideologischen Gründen - nicht die Konsequenzen aus der konstatierten Herausbildung eines antiegalitären beziehungsweise undemokratischen Liberalismus.

Einzig Deneen weist auf die selbstzerstörerische Tendenz des individualistischen Liberalismus (den er verkürzt mit dem Liberalismus gleichsetzt), seine unintendierten antiegalitären Effekte und auch das verkürzte individualistische Freiheitsverständnis hin. Seine Antworten erklären sich jedoch weniger aus seiner treffenden Analyse als aus seinen politischen Präferenzen: Anstatt, wie von Deneen selbst 
gefordert, das ideologische Zeitalter zu überwinden, zielt sein Vorhaben darauf, eine (die liberale) durch eine andere (konservativ-kommunitäre) Ideologie zu ersetzen. Nimmt man die Forderung nach der Überwindung der Ideologie ernst und behauptet also nicht, über die Kenntnis der menschlichen Natur und der ihr entsprechenden, wahren politischen Ordnung zu verfügen, dann kann Gemeinschaft zu fördern jedoch nur bedeuten, Demokratie als Form der Aushandlung gemeinsamer Werte und Überzeugungen ernst zu nehmen und gleiche Freiheit als Voraussetzung dieses Aushandlungsprozesses zu schützen. Wenn aber die liberale Freiheit in ihrer individualistischen Konzeption selbstzerstörerisch ist, weil sie das Ziel der wechselseitigen Garantie gleicher Freiheit aufgrund ihrer antiegalitären Effekte verfehlt, dann lautet die zentrale Frage: Wie muss Freiheit konzipiert sein, damit sie das für ihre wechselseitige Garantie notwendige Erfordernis der Gleichheit erfüllt?

Vor diesem Hintergrund erscheint die von Mounk und Galston vorgenommene analytische Trennung zwischen den Konzepten der Demokratie und des Liberalismus äußerst gewinnbringend und die von ihnen konstatierte Entwicklung hin zu einem undemokratischen Liberalismus einerseits und einer illiberalen Demokratie andererseits treffend. Doch in ihrer Fokussierung auf die Bedrohung des Rechtsstaats durch den Populismus (und damit die illiberale Demokratie) zeigt sich nicht nur ein liberaler Überlegenheitsgestus, sie scheinen auch Ursache und Wirkung der Krise zu verwechseln. Soziale Ungleichheit, Digitalisierung und Migration sind keineswegs, wie hier unterstellt, Ursachen der Krise, sie sind vielmehr Symptome der angesichts eines entfesselten Kapitalismus ungebremst sich bahnbrechenden antiegalitären Effekte des individualistischen Liberalismus oder anders: eines undemokratischen Liberalismus. Babones, der als einer der wenigen hier besprochenen Autoren die globale Dimension mitdenkt, benennt prägnant das eigentliche Problem und seine praktische Ursache: die Gefährdung der Demokratie durch einen neuen liberalen Autoritarismus einer globalisierten Ökonomie und ihrer Experten. Allerdings muss man hier differenzieren: Der globale Kapitalismus selbst ist nicht Ursache der Krise; die dem individualistischen Liberalismus inhärenten antiegalitären Effekte kommen unter seinen Bedingungen ,nur' ungezügelt zum Ausdruck, weil sich die ökonomische Ungleichheit nicht länger durch nationalstaatliche Mechanismen einhegen lässt und sich so ungehindert in politische Ungleichheit übersetzt. Erst vor dem Hintergrund des globalen als eines entfesselten Kapitalismus offenbart sich die dem individualistischen Liberalismus inhärente selbstzerstörerische Wucht. Das Aufkommen rechtspopulistischer Parteien ebenso wie transnationaler Protestbewegungen kann gleichermaßen als Folge dieser Entwicklung verstanden werden. Beide verfolgen ihrem Selbstverständnis nach dasselbe Ziel, wenn auch mit einer völlig anderen politischen Intention: die Demokratie vor dem liberalen Autoritarismus einer globalisierten Ökonomie zu schützen und sie zu stärken. Der entscheidende Unterschied besteht darin, dass die transnationalen Protestbewegungen Liberalismus und Demokratie nur neu ausbalancieren wollen, während die Rechtspopulisten die liberale Rahmung der Demokratie als solche infrage stellen und damit das Problem nicht lösen, vielmehr trachten sie die Disbalance (umgekehrt) einseitig aufzulösen.

Die Gefahr der elitären Tendenz, der Herausbildung eines undemokratischen Liberalismus beziehungsweise der Etablierung einer verstecken Oligarchie oder gar eines liberalen Autoritarismus liegt jedoch nicht in der liberalen Rahmung (bezie- 
hungsweise rechtlichen Beschränkung) der Demokratie als solcher, sondern in dem dieser Rahmung zugrundeliegenden individualistischen Freiheitsverständnis. Die liberalen Demokratien westlicher Prägung basieren auf dem Gedanken, dass der freiheitliche Ausgleich individueller Interessen zum Gemeinwohl führt und die Mitwirkung aller zugleich die Freiheit gewährleistet. Der Umstand, dass manche Interessen gar nicht gehört werden, während andere überproportional großen Einfluss auf politische Entscheidungen haben, zeigt, dass dieser Interessenausgleich als Garantie der Freiheit nicht funktioniert. Grayling weist insofern mit der Dominanz partikularer Interessen in politischen Entscheidungen auf das zentrale Moment der Gefährdung der liberalen Demokratie beziehungsweise - wenn auch von ihm so nicht benannt - der individuellen Freiheit durch ihre eigenen antiegalitären Effekte hin. Er nennt auch klar die Zielvorgabe, nämlich: den Schutz der liberalen Demokratie vor einer Tyrannei der Mehrheit ebenso wie vor einer versteckten Oligarchie. Seine Lösungsansätze aber befördern mit der Betonung der Bildung die elitäre Tendenz eher, als sie $\mathrm{zu}$ verhindern und gehen so an der Ursache vorbei: Die individualistische Konzeption der Freiheit unterminiert das Versprechen der liberalen Demokratie nach gleicher Freiheit, indem sie es einseitig zugunsten der individuellen Freiheit einiger auflöst und so der im Liberalismus angelegten elitären Tendenz zum Durchbruch verhilft.

Der Liberalismus beruht erkenntnistheoretisch auf der Annahme, dass der Mensch zwar einerseits qua Vernunft die Notwendigkeit der Machtteilung erkennt, die konkrete politische Zusammenarbeit aber andererseits vor dem Machttrieb der Einzelnen geschützt werden muss. Deshalb ist die liberale Herrschaft eine entpersonalisierte Herrschaft, es sind nicht Menschen, sondern Verfahren und Institutionen wie Gewaltenteilung oder verfassungsrechtlich garantierte Grundrechte, die den Machtmissbrauch verhindern sollen. Es sind aber nicht nur Menschen, die die Verfahren und Institutionen entwerfen - die liberalen Theoretiker -, sondern auch Menschen, die diese Institutionen und Verfahren am Laufen halten. So übersetzt sich die Spaltung zwischen Vernunft und (Macht-)Trieb zwangsläufig in eine Spaltung zwischen eine die liberalen Ideale schützende, vernünftige Elite und eine von dieser Elite angeleitete, zur Irrationalität neigende Masse. Dem Liberalismus liegt, wie jeder Ideologie, die Annahme einer höheren Wahrheit und damit eine elitäre Tendenz zugrunde, die auf einer Spaltung zwischen den um die jeweilige Wahrheit Wissenden und den (noch) Unwissenden beruht. Im Falle des Liberalismus gerät die auf der ideologischen Wahrheit beruhende Spaltung zwischen Herrschenden und Beherrschten in Widerspruch zu dessen eigener Forderung nach gleicher Freiheit, zu deren Umsetzung er deshalb notwendig eine Verbindung mit der demokratischen als egalitären Herrschaftsform eingehen muss.

Im Rahmen der repräsentativen Demokratie wird die dem Liberalismus inhärente Spaltung neutralisiert beziehungsweise in eine nur temporäre Spaltung zwischen Repräsentanten als Sachverständige der Politik und Repräsentierten als die Masse der durch diese Politik Behandelten übersetzt, sodass politische Gleichheit durch den potenziellen Wechsel zwischen Regierenden und Regierten garantiert wird. Das Problem entsteht, weil der für die repräsentative Demokratie zentrale Wechsel zwischen Regierten und Regierenden unter den Voraussetzungen eines individualistischen Liberalismus durch die aus der unterschiedlichen Nutzung der individuellen 
Freiheit resultierende soziale und ökonomische Ungleichheit ausgehebelt wird. Wird die soziale und ökonomische Ungleichheit nicht - wie dies lange Zeit der Fall war durch wohlfahrtstaatliche Maßnahmen abgemildert (wenn auch nicht gelöst), kommt der ideologische Charakter des Liberalismus zum Tragen, der entgegen der eigenen Forderung nach gleicher Freiheit die Herrschaft einer vermeintlich vernünftigen Elite begünstigt, zuungunsten der demokratischen Mitbestimmung der vermeintlich Unvernünftigen. Die aus dem individualistischen Liberalismus notwendig folgende soziale und ökonomische Ungleichheit übersetzt sich dann in politische Ungleichheit.

Trotz der vor diesem Hintergrund berechtigten Kritik der Populisten geht ihre (mehr oder weniger offene) Forderung nach einer illiberalen Demokratie fehl. Insofern demokratische Herrschaft mit der Entscheidung der Mehrheit selbst ein ideologisches Moment enthält, kann die Konsequenz aus dem Übergewicht des liberalen Elements nicht sein, die liberale (beziehungsweise rechtliche) Rahmung der liberalen Demokratie als solche infrage zu stellen. In ihrer Reinform ist die Demokratie mit dem Problem der Tyrannei der Mehrheit konfrontiert, die wiederum die Herrschaft der einen (Vielen) über die anderen (Wenigen) bedeutet. Noch einmal wird hier deutlich: Liberalismus und Demokratie sind nicht zufällig, nicht nur historisch, vielmehr ideell verbunden. Weil die demokratische Entscheidung gleiche Freiheit voraussetzt, muss diese selbst der demokratischen Entscheidung entzogen sein. Umgekehrt ist gleiche demokratische Teilhabe nicht nur Ausdruck, sondern auch Garantie der gleichen individuellen Freiheit. Politische Gleichheit ist ein notwendiges Erfordernis der Freiheit als ein Recht, das wir uns wechselseitig garantieren.

Die Herausforderung scheint vor dem Hintergrund des Gesagten darin zu liegen, Freiheit neu zu denken. Will man die Freiheit eines jeden gleichermaßen schützen, sodass sich die ideologische Versuchung der Demokratie (Tyrannei der Mehrheit) und des Liberalismus (Autoritarismus der Vernünftigen) gegenseitig weitestgehend neutralisieren, ist es unabdingbar, die negative Freiheit um eine soziale Dimension $\mathrm{zu}$ erweitern. Geschützt werden muss nicht nur der individuelle Bereich vor staatlicher Willkür, geschützt werden muss auch die politische Zusammenarbeit vor individueller Willkür. Denn wenn individuelle (oftmals ökonomische) Interessen einiger Weniger die politischen Entscheidungen dominieren, dann gefährdet das langfristig die individuelle Freiheit aller. Soziale Freiheit schützt indirekt die individuelle Freiheit, jedoch nicht die individuelle Freiheit einiger, sondern die gleiche individuelle Freiheit aller! Für einen um eine soziale Dimension der Freiheit erweiterten politischen Liberalismus ist das kapitalistische Wirtschaftssystem nicht im selben Maße sakrosankt wie für den individualistischen Liberalismus, es ist vielmehr selbst Gegenstand demokratischer Aushandlung und steht folglich unter dem Vorbehalt sozialer Freiheit. Auf globaler Ebene fehlen dafür bislang die Strukturen, hier muss es allererst darum gehen, der globalisierten Ökonomie politisch etwas entgegenzusetzen; auf nationalstaatlicher Ebene wäre mit Blick auf die soziale (als Voraussetzung der individuellen) Freiheit bereits viel gewonnen, wenn die Tätigkeit von Lobbygruppen, Parteispenden, die Verknüpfung von politischem Amt und Posten in der Wirtschaft und ähnliches dahingehend reglementiert, kontrolliert oder verboten würde, dass der Einfluss von organisierten Einzelinteressen auf politische 
Entscheidungen so weit wie möglich ausgeschlossen wird. Nur ein demokratischer Liberalismus, der die individuelle wie die soziale negative Freiheit gleichermaßen garantiert, kann der liberalen Forderung nach gleicher Freiheit gerecht werden, anstatt wie in seiner individualistischen Form zur Ideologie zu erstarren und dadurch die Demokratie und zugleich seine eigene Existenzgrundlage zu untergraben.

\section{Auswahlbibliografie}

- Albright, Madeleine: Faschismus. Eine Warnung, 320 S., Du Mont, München, 2018 (engl. 2018).

- Babones, Salvatore: The New Authoritarianism. Trump, Populism, and the Tyranny of Experts, 120 S., Polity Press, Cambridge, 2018.

- Brennan, Jason: Gegen Demokratie. Warum wir die Politik nicht den Unvernünftigen überlassen dürfen, 464 S., Ullstein, München, 2017 (engl. 2016).

- Brock, Ditmar: Die überdehnte Staatlichkeit. Warum der Westen scheitern könnte, 337 S., Springer, Frankfurt a. M., 2018.

- Deneen, Patrick J.: Why Liberalism Failed, 224 S., Yale UP, New Haven, CT/ London 2018.

- Ferguson, Niall: Der Niedergang des Westens. Wie Institutionen verfallen und Ökonomien sterben, 208 S., List, Berlin, ${ }^{2} 2014$ (engl. 2012).

- Galston, William A.: Anti-Pluralism. The Populist Threat to Liberal Democracy, 176 S., Yale UP, New Haven, CT/London 2018.

- Grayling, A. C.: Democracy and Its Crisis, 240 S., Oneworld, London, 2018 (orig. 2017).

- Levitsky, Steven/Ziblatt, Daniel: Wie Demokratien sterben. Und was wir dagegen tun können, 320 S., DVA, München, 2018 (engl. 2018).

- Mahbubani, Kishore: Has the West Lost It? A Provocation, 106 S., Penguin, New York 2018.

- Mounk, Yascha: Der Zerfall der Demokratie. Wie der Populismus den Rechtsstaat bedroht, 368 S., Droemer Knaur, München, 2018 (engl. 2018).

- Runciman, David: How Democracy Ends, 256 S., Profile Books, London, 2018.

- Snyder, Timothy: Der Weg in die Unfreiheit. Russland, Europa, Amerika, 376 S., Beck, München, 2018 (engl. 2018).

- Wiatr, Jerzy J. (Hrsg.): New Authoritarianism. Challenges to Democracy in the 21st Century, 184 S., Budrich, Opladen u. a. 2019.

Funding Open Access funding provided by Projekt DEAL.

Open Access Dieser Artikel wird unter der Creative Commons Namensnennung 4.0 International Lizenz veröffentlicht, welche die Nutzung, Vervielfältigung, Bearbeitung, Verbreitung und Wiedergabe in jeglichem Medium und Format erlaubt, sofern Sie den/die ursprünglichen Autor(en) und die Quelle ordnungsgemäß nennen, einen Link zur Creative Commons Lizenz beifügen und angeben, ob Änderungen vorgenommen wurden.

Die in diesem Artikel enthaltenen Bilder und sonstiges Drittmaterial unterliegen ebenfalls der genannten Creative Commons Lizenz, sofern sich aus der Abbildungslegende nichts anderes ergibt. Sofern das betreffende Material nicht unter der genannten Creative Commons Lizenz steht und die betreffende Handlung 
nicht nach gesetzlichen Vorschriften erlaubt ist, ist für die oben aufgeführten Weiterverwendungen des Materials die Einwilligung des jeweiligen Rechteinhabers einzuholen.

Weitere Details zur Lizenz entnehmen Sie bitte der Lizenzinformation auf http://creativecommons.org/ licenses/by/4.0/deed.de.

Frauke Höntzsch ist Wissenschaftliche Mitarbeiterin am Lehrstuhl für Politikwissenschaft/Politische Theorie der Universität Augsburg. Sie wurde zum Thema „Individuelle Freiheit zum Wohle Aller. Die soziale Dimension des Freiheitsbegriffs im Werk des John Stuart Mill“ promoviert. Neben dem (Sozial-)Liberalismus/der liberalen Demokratie forscht sie zur politikwissenschaftlichen Anthropologie, zu Widerstand (und Terrorismus) und zu den Menschenrechten. 